\title{
Method of the Year 2016: Epitranscriptome analysis
}

\author{
Chemical modifications on ribonucleotides are being profiled with increased efficiency and \\ appreciated as important regulatory features.
}

In 2006 Andrew Fire and Craig Mello were awarded the Nobel Prize in Physiology and Medicine for their discovery of RNA interference. Their findings triggered a flood of research into the function of noncoding RNAs that continues to this day. One emerging question is how RNA molecules themselves are regulated; more specifically, what is the function of post-transcriptional modifications found on all RNA species? Recent technological breakthroughs, driven mainly by sequencing-based approaches, have enabled epitranscriptome analysis-the genome-wide profiling of such RNA modifications-and already point to important functional roles of the epitranscriptome (see News Feature p15).

Before proceeding, let us first clarify terms. We use the literal definition of the Greek prefix 'epi' (above) in epitranscriptomics and consider any modification added to the ribonucleotides irrespectively of known function or heritability.

For decades little attention was paid to RNA modifications - the discovery of marks on RNA dates back to the 1960s and 1970s, but they were thought to be confined to tRNAs and rRNAs, and research focused on epigenetic marks on DNA. The discovery that chemical marks occur in all RNA species, together with reports of writers and erasers that dynamically add and remove these marks, led to renewed interest in RNA modifications. For example, the link between an enzyme that removes a methyl group from adenosine and Alzheimer's disease risk suggests an important regulatory role for the modification in neuronal health. A Commentary by Arne Klungland and colleagues on p18 discusses the role of writers and erasers of methyl marks on adenosine in meiosis and pluripotency.

A link between RNA modifications and cancer has prompted the NIH to issue an announcement of a development research grant for evaluating the role of epitranscriptomics in cancer biology with new tools and technologies. One exciting possibility is the targeted modification of RNA marks using the CRISPR system (see Methods to Watch p33).

Overall though, functional characterization is still in its infancy; and in order to understand what these marks do, one must first determine their abundance and location.

There are over one hundred different known modifications on RNA - for a comprehensive look we recommend Modomics, a database that lists all known modifications. And yet, the field is still in its discovery phase, with new methods being developed to discover and catalogue modifications. A Review on p23 by Chengqi Yi and colleagues describes the latest methods to detect the most common modifications, such as methyl-6-adenosine, pseudouridine and inosine.

Further development is still needed, and experts have refreshingly diverse views on how to best tackle the current bottlenecks. Many methods rely on antibody-based enrichment, but antibodies can be plagued by nonspecificity. Some researchers therefore focus on new sequencing technologies, such as nanopores or PacBio's single-molecule sequencing strategy to provide direct readouts for modifications.

For other marks, such as pseudouridine, better chemical labeling strategies for enrichment could provide a more comprehensive picture. Researchers exploit the fact that, during cDNA synthesis, reverse transcriptase stalls and often stops when encountering certain chemical groups on the nucleotides. The resulting unique read signatures can be the foundation for profiling, and with the right software more than one type of modification could be probed at once. Yet other marks, such as adenosine-to-inosine editing, bring about codon changes that can be profiled with the appropriate computational approaches. It is unlikely that there will be a one-size-fits-all method; and a multipronged approach looks to be most fruitful to comprehensively catalogue the chemical landscape present on RNA. Larger questions, like that of heritability, will also have to be tackled.

Despite gaps in our knowledge and the mountain of work ahead, it is clear that impressive headway has been made to chart rough maps of the RNA landscape. For our subjective selection of other areas to keep an eye on in the coming year, see our Methods to Watch section (p32-35).

We wish all our readers a happy 2017. 